\section{AIDS and malaria experiments}

SIR - Charles Gilks (Nature 354, 262; 1991) suggests that AIDS may have initially entered the human population by direct inoculation into human prisoner volunteers with malaria infected blood which may have been contaminated with the primate precursors of the human AIDS virus. Gilks states “... my theory is testable. The records of human volunteer experiments, particularly those involving prisoners in the United States, can be checked to see if any further, unpublished studies took place." But Gilks' theory is not testable, and will ultimately be added to the increasing list of unproven theories proposed to explain the origin of the AIDS epidemic.

As a clinical associate at the National Institutes of Health (NIH) in 1962, I and my colleagues performed monkey and human malaria studies in prisoner volunteers at the Atlanta penitentiary, discovering the indirect fluorescent antibody test for malaria (for example, S. F. K. et al. Science 135, 1130; 1962). All research programmes at the penitentiary had to receive prior approval from the NIH ethical behaviour board, which never allowed direct primate blood inoculation into humans. Our experiments always and only involved mosquito transmission of monkey and human malaria into volunteers. It is a matter of scientific fact that AIDS cannot be transmitted by mosquitoes or other arthropods.

Gilks' suggestion that "material from several of the original experiments could still exist and could be tested for the presence of retroviruses" is unfortunately untrue. In the case of the prisoner volunteer studies carried out at the Atlanta penitentiary up to 1962 serum samples from the volunteers, to the best of my inquiries, no longer exist after over a 30-year lapse. Blood from monkeys infected with Plasmodium gonderi used in prisoner volunteer studies in 1961 and 1966 by other investigators still does exist at the Centers for Disease Control. But, as in our studies, these infected $P$. gonderi monkey blood samples were used only in failed attempted mosquito transmission studies, never by direct human inoculation, which has always been prohibited in the United States.

SANFORD F. KUVIN

44 Cocoanut Row,

Palm Beach, Florida 33480, USA

SIR - Gilks ${ }^{1}$ has offered a provocative explanation for the origin of the human immunodeficiency viruses in which researchers may have encouraged animal retroviruses to jump the species gulf by injecting simian blood into humans, including themselves. If a long and possibly fruitless search for surviving evi- dence is warranted on the strength of his arguments, we must also consider alternative explanations for the origin of HIV involving clinical research.

After the First World War moderate professional opinion was deeply divided over the question of whether the physical and mental disabilities of mid- and late life could be postponed or averted by boosting sex-hormone production. Among the treatments offered for rejuvenation, testicular grafts were regarded as particularly valuable and effective, hundreds having been performed in Europe and the United States by the mid-1920s $\mathrm{s}^{2-4}$. There were always more willing patients than donor organs from healthy young men and, as knowledge of transplant biology was primaeval, surgeons frequently used animal testicles, preferably from apes and monkeys.

Common chimpanzees were favoured donors, not only because of a genetic affinity with man but also on account of their impressively large testicles. Few details have survived about the history and condition of these animals or the much larger numbers of monkeys, although many had probably originated in the French colonies of West Africa. One testicle was removed, sliced and grafted either to the abdominal rectus muscle or to join the organs resident in the patient's scrotal pouch. In either case, the grafts effectively inoculated him with whatever infectious agents they were carrying. When synthetic testosterone became available in 1935 and careful testing showed that rejuvenation therapy had been wishful thinking, testicular grafting became disreputable and former patients threatened their doctors with suits. Nevertheless, administration of animal sex organs by grafting or injection did not completely die out.

Although we may never know precisely how many people received simian testicular grafts they far outnumber those treated with malarial blood. But by drawing attention to the possibility of a biomedical origin of HIV, however speculatively, we risk the possibility of creating new scapegoats. Many of the rejuvenators were well-intentioned, if misguided, doctors and it would be regrettable if they or the malaria researchers were to be charged with the grave responsibility for the AIDS epidemic on the basis of circumstantial evidence.

Department of Physiology,

ROGER G. GOSDEN

University of Edinburgh, Edinburgh EH8 9AG, UK

1. Gilks, C. Nature 354, 262 (1991).

2. Stanley, L. L. Endocrinology 6, 787-794 (1922)

3. Hoskins, R. G. Endocrinology 9, 277-296 (1925).

4. Hamilton, D. W. The Monkey Gland Affair (Chatto and Windus, London, 1986).
Neutral terms

SIR - Woese et al. ${ }^{1}$ claim that archaebacteria's most recent common ancestor appears to have been with eukaryotes, not with eubacteria. This stems from rooting the ribosomal RNA tree in the eubacterial branch by comparisons of two pairs of paralagous protein genes. The reason is that each archaebacterial protein in the reported studies has greater similarity to its eukaryotic than to its eubacterial homologues. But several examples of the opposite exist. Furthermore, a eubacterial ATPase isolated from the eubacterium Thermus thermophilus ${ }^{2}$ more resembles the archaebacterial and eukaryotic ATPases than the Escherichia coli ATPase cited by Woese et al. More strikingly, an archaebacterial glutamate dehydrogenase specifically related to its homologue from 'higher' eukaryotes, whereas its eubacterial homologue is related to 'lower' eukaryotes ${ }^{3}$. This indicates that protein phylogenetic trees cannot be used at the moment to root with confidence the ribosomal RNA tree.

Two possibilities remain: either the root is in the eubacterial or archaebacterial branch and the last common ancestor was prokaryotic-like, or the root is in the eukaryotic branch and it resembled more proto-eukaryotes. The first hypothesis fits with the theory that life originated at high temperature, as the common ancestor of all prokaryotes was probably a thermophile ${ }^{1}$, but the second fits better with the antiquity of introns and the 'RNA world' hypothesis. I suggest that prokaryotes arose as a specific adaptation to thermophily by reductive evolution from a common mesophilic ancestor to pro- and eukaryotes.

Both the traditional nomenclature ${ }^{4}$ and that proposed by Woese et al. are biased in favour of the first hypothesis. The terms 'pro' and 'eu' suppose that prokaryotes are primitive compared to eukaryotes, and the term 'archaea' suggests that archaebacteria are the more ancient life forms. We should be looking for nomenclatures which avoid answering unsolved questions. Otherwise, if prokaryotes turn out to have originated from primitive eukaryotes by reductive evolution, we will have to call them postkaryotes! I therefore propose the neutral terms akaryotes (without nucleus) and synkaryotes (with nucleus) for a natural classification without prejudice.

PATRICK FORTERRE

Institut de Genetique et Microbiologie,

Université Paris-Sud, Bâtiment 409,

91405 Orsay Cedex, France 1. Woese, C. R. et al. Proc. natn. Acad. Sci. U.S.A. 87,
$4576(1990)$.
2. Yokohama, K. J. biol. Chem. 265, $21940-21950(1990)$.
3. Benachenhou, N. \& Baldacci, G. Molec. gen. Genet. (in
the press).
4. Mayr, E. Nature 348, 491 (1990). 